\title{
Molecular characterization of an adenoviral vector resulting from both homologous and nonhomologous recombination
}

\author{
Jason G. Smith and Stephen L. Eck \\ Division of Hematology/Oncology, Department of Medicine, University of Pennsylvania Medical Center, \\ Philadelphia, Pennsylvania 19104.
}

Recombinant adenoviral vectors are being used extensively for gene transfer. During the construction of an E1-deleted virus expressing the human B7-1 gene, an aberrant recombinant (Ad.ihB7-1) arose with an unusual 5' sequence. Characterization and sequencing of Ad.ihB7-1 showed that its structure was the result of both homologous and nonhomologous events. The most striking features of the construct were the incorporation of bacterial genomic DNA, an additional inverted terminal repeat, and portions of E1a. The appearance of this construct has implications for vector design and indicates the need for careful analysis and characterization of recombinant adenoviral vectors for clinical use.

Key words: Adenoviral vectors; recombination; transgenes.

$\mathrm{R}$ ecombinant mammalian viruses have been shown to be effective vectors for gene transfer and are now being tested in human clinical applications. ${ }^{1}$ Viruses have evolved efficient means of DNA encapsidation, cell entry, and gene expression. Furthermore, modifications can be made to render them suitable and safe for human gene therapy applications. A number of viruses have been engineered for use as gene therapy agents, including herpes simplex virus, human and murine retroviruses, adeno-associated virus, vaccinia virus, and adenovirus. ${ }^{2}$

Recombinant adenoviral vectors (rAds) offer a number of advantages over other viral vectors. They are able to transduce both dividing and nondividing cells, have very low pathogenicity, can be prepared in high titers, efficiently transduce a wide range of cells, and can be easily manipulated. ${ }^{3}$ The rAds used in human applications are typically E1-deleted, E3-deleted, or both, with the transgene usually positioned in E1. ${ }^{4}$ E1-deleted viruses are replication-defective and must be propagated on cell lines that can transcomplement for these missing functions (e.g., 293 cells). Newer generations of rAds have more extensive deletions or alterations of E2 or E4. ${ }^{5}$

rAds are produced by homologous recombination between a plasmid that carries the transgene and associated regulatory elements and a viral genome that has been altered previously (e.g., alterations or deletions in E2, E3, or E4). The viral genome used in the cotransfection is further altered to prevent its replication in the

Received May 5, 1998; accepted November 1, 1998.

Address correspondence and reprint requests to Dr. Stephen L. Eck, Division of Hematology/Oncology, University of Pennsylvania Medical Center, BRB-III Room 512, 421 Curie Boulevard, Philadelphia, PA 19104-6160. E-mail address: ecks@mail.med.upenn.edu

(C) 1999 Stockton Press 0929-1903/99/\$12.00/+0 absence of recombination with plasmid DNA. This is often accomplished by removal of the $5^{\prime}$ inverted terminal repeat (ITR) and packaging sequence by restriction digestion. Recombination between the viral genome and plasmid DNA replaces portions of the viral genome with plasmid DNA containing the transgene and restores the packaging sequence in the rAd construct. ${ }^{6}$

This method of producing rAds is effective; however, it allows for the possibility of producing unwanted recombinant viruses at several points during production. The use of cell lines to transcomplement deleted gene products (e.g., E1 and E4) during propagation of the replication-defective rAd is necessary but imposes a growth disadvantage for rAds compared with wild-type (wt) viruses or replication-competent adenoviruses (RCAs). The copy number of transcomplementing genes in cell lines (e.g., E1 gene in 293 cells) is lower than the copy number of these genes in cells infected with RCA or wt virus. Therefore, production of these gene products may become rate-limiting for rAd propagation. RCA and wt virus do not have this restriction and, therefore, have a growth advantage over rAds. Contamination of rAd stocks by RCA or wt virus will result in increasingly higher proportions of contaminant over rAds during serial passage due to this growth advantage. $^{7}$

The ability of adenoviral DNA to undergo homologous recombination events may be a product of the mechanism of viral DNA replication. Adenovirus replication produces single-strand templates that may invade duplexes, ejecting the resident strand. The recombinatorial intermediates are then resolved by host cellular enzymes, resulting in recombinant products. ${ }^{8}$ The ease with which viral DNA recombines may allow unwanted recombinations in addition to those intended to produce 
the desired rAd. The transcomplementing viral genes present in the propagating cell line may contain additional flanking viral DNA. For example, the 293 cell line, which is the most commonly used packaging cell line, was created by transfecting randomly sheared viral DNA into human embryonic kidney cells. ${ }^{9}$ This resulted in the incorporation of 4.3 kilobases $(\mathrm{kb})$ of the $5^{\prime}$ end of adenovirus type 5 (Ad5), including the ITR, E1a, E1b, and part of protein IX into the cellular genome. ${ }^{10}$ Although only the E1 sequences are needed to support the propagation of E1-deleted rAds, the additional viral DNA in the cellular genome provides areas for recombination with the rAd. The potential consequences of these unwanted recombinations are the formation of RCAs, loss of transgene function, or introduction of nonfunctional DNA. The "ideal" transcomplementing cell line for E1-deleted rAd would contain only E1a and E1b.

The rAd may be further disadvantaged by alterations of the genome size, which lead to inefficient packaging of rAd DNA into capsids. The intact Ad5 genome is $36 \mathrm{~kb}$ in length. Viable mutants have been constructed with lengths that are $\leq 105 \%$ of the wt genome. Recombinants with larger inserts grow slowly, if at all, and are unstable. ${ }^{11}$ This can lead to a greater disparity in the growth rate of rAds compared with wt virus or RCA. The lower size limit for efficient packaging has also been described previously. ${ }^{12}$ Parks and Graham ${ }^{12}$ demonstrated a lower packaging limit of $\sim 75 \%$ of the wt genome. Constructs over this size limit grow equally well; genome lengths below this limit grow poorly if at all.

We have characterized a rAd with an unusual $5^{\prime}$ sequence that arose during the construction of an E1, E3-deleted adenoviral vector in 293 cells. The viral genome has been analyzed in detail to elucidate the mechanism by which it arose. This recombinant virus seems to be the product of both homologous and nonhomologous recombination events. The formation of this rAd demonstrates the instability of rAd genomes and the need to carefully characterize adenoviral vectors intended for human clinical trials.

\section{MATERIALS AND METHODS}

\section{Construction of plasmids and rAds}

The plasmid pAdCMVlink has been described previously. ${ }^{13}$ Briefly, this plasmid consists of a multiple cloning site downstream of the cytomegalovirus (CMV) immediate early enhancer/promoter element in a plasmid containing portions of the $5^{\prime}$ region of Ad5. A reverse transcriptase-polymerase chain reaction (PCR)-generated 958-base pair (bp) product of human B7-1 (hB7-1) cDNA was cloned into the pUC19 plasmid (pUC19hB7-1) and sequenced to confirm the identity of the PCR product. pAdCMVhB7-1 was constructed by cloning a Bam HI/BglII restriction fragment of pUC19hB7-1 into the multiple cloning site of pAdCMVlink at the BglII restriction site (see Fig 3). The mouse B7-1 cDNA was excised from pLL218 (a gift of Dr. Lewis Lanier, DNAX, Palo Alto, Calif) and subcloned into pUC19. pAdCMVmB7-1 was constructed by ligating a $B g l \mathrm{II} / \mathrm{ClaI}$ restriction fragment of pUC19mB7-1 into the large $B g l \mathrm{II} / \mathrm{ClaI}$ restriction fragment of pAdCMVlink.

Ad.ihB7-1 was constructed by coprecipitating pAd-CM-
VhB7-1, which was linearized by digestion with NheI, and d $17001^{14}$ adenoviral genomic DNA, which was first digested with ClaI to remove the 5' ITR and packaging sequence, into 293 cells (American Type Culture Collection (ATCC), Manassas, Va). Ad.mB7-1 was constructed by the same method using pAdCMVmB7-1. Ad.lacZ consists of the $\beta$-galactosidase $(\beta$-gal) gene under the control of a CMV-enhanced chicken $\beta$-actin promoter positioned in place of E1a and part of E1b in a partially E3-deleted viral genome, sub360. ${ }^{15}$

\section{Flow cytometry}

The $h B 7-1$ was detected using a primary mouse monoclonal antibody against human BB1/B7 (Becton Dickinson, San Jose, Calif) and a secondary fluorescein isothiocyanate-conjugated, antigen-binding, fragment-specific antibody against mouse immunoglobulin G (Sigma, St. Louis, Mo). $\beta$-gal was detected with the substrate fluorescein di- $\beta$-D-galactopyranoside (Molecular Probes, Eugene, Oreg) following the manufacturer's recommended protocol. Analysis was performed on a FACScan flow cytometer (Becton Dickinson).

\section{Analysis of Ad.ihB7-1 for replicative ability}

HeLa cells (ATCC) were cultured to near confluency in six-well dishes. Duplicate wells were transfected at a multiplicity of infection (MOI) of 50 based on plaque-forming units with dl7001, Ad.ihB7-1, or Ad.lacZ. After 2 days of incubation, one well from each transfection was analyzed by flow cytometry for either hB7-1 or lacZ as described above. The second well was harvested on day 3 and subjected to three cycles of freezing and thawing in $1 \mathrm{~mL}$ of $10 \mathrm{mM}$ tris(hydroxymethyl)aminomethane. Cell debris was removed by centrifugation, and $50 \mu \mathrm{L}$ of lysate from each transfection was used to transfect duplicate wells of a new six-well dish of HeLa cells.

\section{Sequencing of Ad.ihB 7-1 and pAdCMVhB7-1 and sequence analysis}

Sequencing primers for Ad.ihB7-1 and pAdCMVhB7-1 were designed using MacVector DNA analysis software (Oxford Molecular, Oxford, UK). The position of the primers and the direction of sequencing are indicated in Figures 2 (Ad.ihB7-1) and 3 (pAdCMVhB7-1). Ad.ihB7-1 was digested by restriction endonuclease SpeI. DNA fragments were separated by electrophoresis in a $1 \%$ agarose gel. A 4-kb fragment was purified by ion exchange chromatography (Geneclean III, Bio 101, Vista, Calif) before sequencing. pAdCMVhB7-1 was grown in Escherichia coli strain DH5 $\alpha$ (Life Technologies, Gaithersburg, Md) and purified by ion-exchange chromatography (Qiagen, Santa Clarita, Calif). All sequencing was done by the DNA Sequencing Facility of the Department of Genetics and Cancer Center of the University of Pennsylvania using a 377 or 373A Stretch sequencer (Applied Biosystems, Burnsville, Minn). Sequence analysis including open reading frame (ORF) determination was performed using MacVector DNA analysis software (Oxford Molecular).

\section{PCR}

Two oligonucleotides (F1, 5'-CGGTGGATTATGCGGCAATGAACG-3'; B1, 5'-TGTCGGAACAGGCAAAAAACGGACG$\left.3^{\prime}\right)$ were synthesized (CyberSyn, Lenni, Penn) that aligned to sequences in the E. coli genomic fragment found in Ad.ihB7-1 (Fig 2) This combination of oligonucleotides when used in a PCR reaction will amplify a segment of DNA that is $403 \mathrm{bp}$ in length. PCR reactions were performed with $30 \mathrm{ng}$ of DNA 


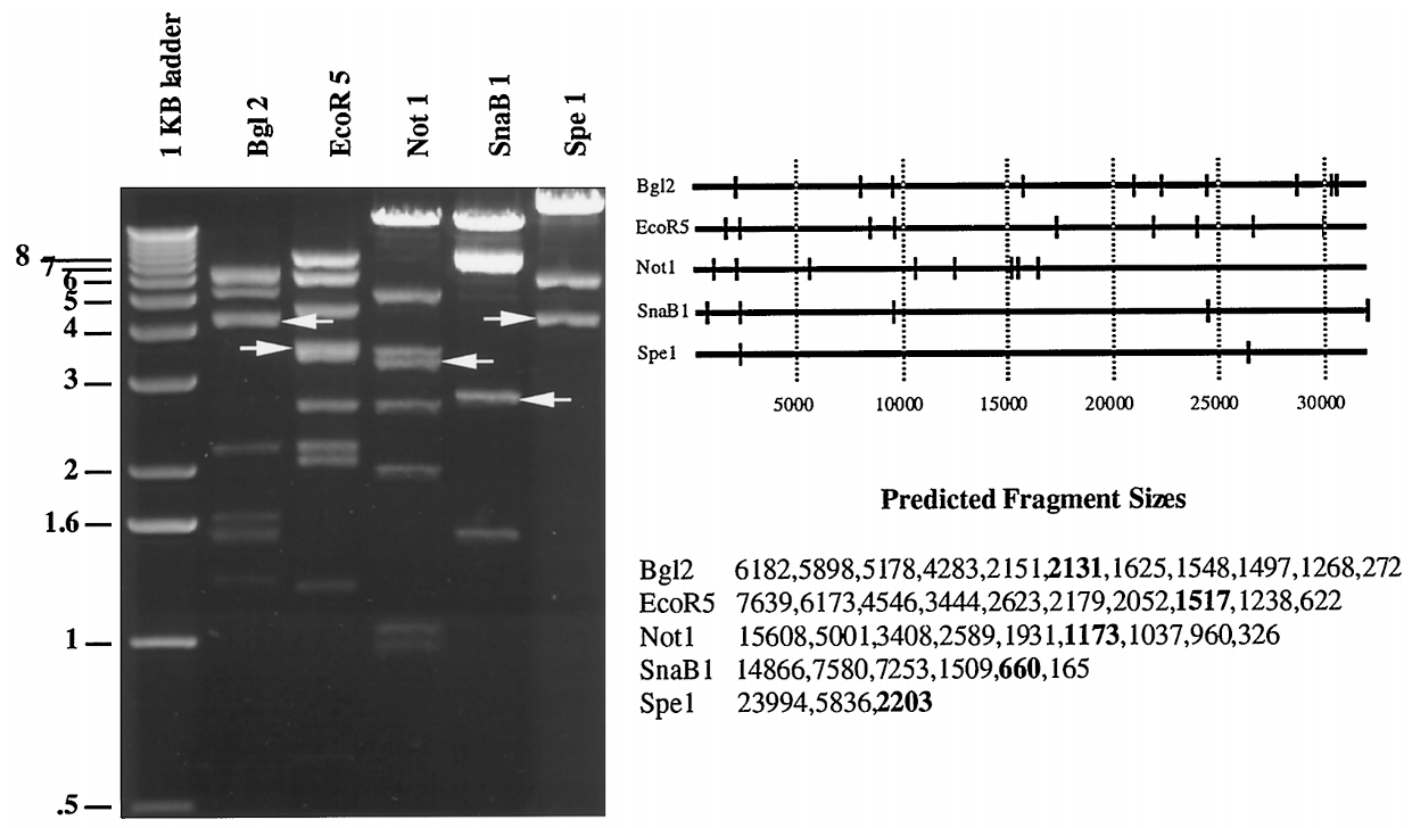

Figure 1. DNA isolated from Ad.inB7-1 was digested with the restriction endonucleases Bg/ll, EcoRV, Notl, SnaBI, and Spel. The location of the digestion sites and fragment sizes based on the predicted sequence of Ad.ihB7-1 are listed. Fragments listed in bold are absent from the ethidium bromide-stained agarose gel. Arrows on the agarose gel indicate additional bands that were not predicted by the sequence of Ad.inB7-1.

using a cycling program of $95^{\circ}$ (30 seconds), $58^{\circ}$ (30 seconds), and $72^{\circ}$ (30 seconds). A total of 30 cycles was performed followed by an additional elongation step at $72^{\circ}$ (5 minutes). All reactions in a given experiment were performed concurrently using the same mixture of enzyme, buffer, $\mathrm{MgCl}_{2}$, and primers with tubes containing water instead of DNA as negative controls for contamination. PCR products were analyzed using agarose electrophoresis.

\section{Southern blot analysis of pAdCMVhB7-1}

pAdCMVhB7-1 was digested with the restriction endonucleases MscI, BglII/NruI,EcoRI/EcoRV,EcoRI/NruI，EcoRI/ SphI, and EcoRI/StuI. Ad.ihB7-1 DNA was digested with SspI as a positive control. Restriction fragments were separated by agarose electrophoresis and transferred to a charged nylon membrane (Boehringer Mannheim Biochemicals, Indianapolis, Ind). The 403-bp PCR product described above was random primer labeled (Prime-It II, Stratagene, La Jolla, Calif) with $\left[{ }^{32} \mathrm{P}\right]$ deoxyadenosine triphosphate and used as a probe. Autoradiograms were developed after 16 and 54 hours of exposure.

\section{Competition assay}

Initially, adenoviral genomic DNA from Ad.mB7-1 and Ad.ihB7-1 was calcium phosphate-precipitated into 293 cells in duplicate dishes in molar ratios of 10:1, 1:1, and 1:10 (320 ng of total DNA per precipitation). Cells were incubated at $37^{\circ} \mathrm{C}$ for 7 days. Cell pellets were lysed by multiple freeze/thaw cycles, cell debris was removed by centrifugation, and adenoviral DNA was obtained by pronase digestion followed by phenol extraction and ethanol precipitation. A total of $10 \mu \mathrm{g}$ of the resulting DNA was digested with restriction endonuclease SpeI. Restriction fragments were separated by agarose electrophoresis and transferred to a charged nylon membrane
(Boehringer Mannheim Biochemicals). A 642-bp restriction fragment of the CMV promoter from pAdCMVlink was random primer labeled (Prime-It II, Stratagene) with $\left[{ }^{32} \mathrm{P}\right]$ deoxyadenosine triphosphate and used as a probe. PhosphorImager data were obtained after 18 hours and analyzed using ImageQuaNT software (Molecular Dynamics, Sunnyvale, Calif).

This experiment was subsequently repeated with $2.2 \mu \mathrm{g}$ of total DNA per precipitation. Separately, $3 \mu \mathrm{g}$ or $9 \mu \mathrm{g}$ of adenoviral DNA from each precipitation was digested with restriction endonuclease SpeI. Restriction fragments were separated by agarose electrophoresis and transferred to charged nylon membranes (Boehringer Mannheim Biochemicals). Positive controls consisting of the original DNA mixed in molar ratios of $1: 10,1: 1$, and $10: 1$ and digested with restriction endonuclease SpeI were used for this experiment. The resulting blots (one each for the set of 3- $\mu$ g and 9- $\mu$ g digests) were probed and analyzed as described previously.

\section{RESULTS}

\section{Construction and functional characterization}

We constructed an E1, E3-deleted rAd containing the hB7-1 gene under the control of the CMV immediate early enhancer/promoter. The rAd was constructed by cotransfecting adenoviral genomic DNA in 293 cells with a complete deletion of E3 (dl7001) and plasmid DNA containing the hB7-1 gene with its associated regulatory elements (pAdCMVhB7-1). The viral DNA was first digested with ClaI at a unique site in E1a (base pair 357 of E1a) to remove the cis-acting packaging element and 5' ITR, thus rendering the genomic DNA incapable of producing virions. The plasmid DNA was 


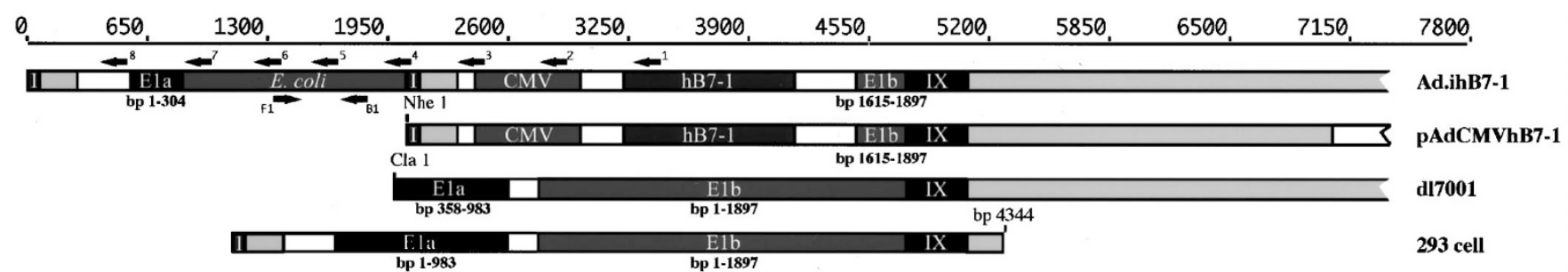

Figure 2. Final sequence of Ad.ihB7-1 with the components of the cotransfection from which it was made (pAdCMVhB7-1 and dl7001) and the Ad5 sequences in the 293 cell genome. I, ITR; $E$. coli, base pairs 129011-130201 of $E$. coli minutes 9-11 (GenBank accession number U82664); CMV, CMV immediate early enhancer/promoter; IX, adenovirus protein IX; and hB7-1, hB7-1 cDNA. Arrows indicate the primers used for sequencing (1-8) or PCR (B1, F1). Primer sequences are: 1, 5'-ATGGTGATGTTCCCTGCCTC-3'; 2, 5'-ATATAGACCTCCCACCGTACACGC-3'; 3, 5'-GCGTTACTATGGGAACATACG-3'; 4, 5' -TCATCCATCCTCTGCTTG-3'; 5, 5' -TTACGCCAGTTTCGTCAC-3'; 6, 5' -GCACTTTGTTCATGCTCTG-3'; 7, 5'-GGGGATGATTGGCTATATG-3'; 8, 5'-AAACGCCAACTTTGACCC-3'; B1, 5'-CGGTGGATTATGCGGCAATGAACG-3'; and F1, 5'-TGTCGGAACAGGCAAAAAACGGACG-3'. In addition, selected restriction enzyme digestion sites are indicated.

linearized using NheI to cut at a unique site $5^{\prime}$ to the ITR prior to cotransfection. Cotransfection of these elements resulted in the replacement of the E1a gene of the virus with the transgene cassette from the plasmid, restoration of the packaging sequence, and replication in 293 cells.

Plaques arising from the initial cotransfection were expanded on 293 cells. The lysate from these expansions was used to infect HeLa cells. Transgene expression (hB7-1) was demonstrated by flow cytometric analysis (data not shown). Plaques that tested positive (4 of 13) were subjected to a second round of plaque purification. The plaque whose expanded lysate resulted in the highest percentage of transduced cells after the second round of plaque purification was expanded and purified into a vector seed stock. Subsequent preparations of virus (Ad.ihB7-1) were made from this original stock.

The ability of Ad.ihB7-1 to express functional hB7-1 was assessed in a T-cell proliferation assay as described previously. ${ }^{16}$ In the presence of a primary signal through the T-cell receptor, T cells exposed to a second, costimulatory signal will proliferate. B7-1 provides this costimulatory signal through binding to the $\mathrm{CD} 28$ receptor on the T cell. Melanoma cells transduced with Ad.ihB7-1 but not Ad.lacZ were capable of stimulating T-cell proliferation, indicating that biologically active hB7-1 was being produced. ${ }^{16}$ Thus, this recombination procedure produced a rAd with the desired functional characteristics.

\section{Determination of sequence and sequence analysis}

After the initial construction and functional characterization of Ad.ihB7-1, restriction digestions using a variety of endonucleases were performed to verify the organization of the rAd (Fig 1). The presence of the transgene was indicated by digestion with NotI. The restriction endonuclease SpeI is expected to digest Ad.ihB7-1 at two locations and should result in a 2-kb piece containing the $5^{\prime}$ region of the vector, a 5.8-kb piece containing the $3^{\prime}$ end, and a 24-kb piece. Digestion with SpeI, however, resulted in the formation of a 4-kb band instead of the $2-\mathrm{kb}$ band. This change in band size (from the predicted sequence) indicated that additional DNA was present at the 5' end of Ad.ihB7-1. Further digestions with the restriction endonucleases BglII, EcoRV, and SnaBI localized this additional sequence to a region $5^{\prime}$ to the hB7-1 cDNA.

The 4-kb SpeI fragment containing the $5^{\prime}$ region of Ad.ihB7-1 was then sequenced from the $5^{\prime}$ end to base pair 89 of the hB7-1 cDNA. Primer sequences and positions are indicated in Figure 2. The entire vector sequence could be identified through comparison with published sequences. The components of Ad.ihB7-1 are depicted in Figure 2 and consist of base pairs 1-863 of Ad5 (including base pairs 1-304 of E1a), base pairs 129011-130201 of E. coli minutes 9-11 (GenBank accession number U82664), and the predicted sequence of NheI-linearized pAdCMVhB7-1. Whereas the total length of the anticipated rAd was $32 \mathrm{~kb}$, the actual construct is $34 \mathrm{~kb}$ in length.

Using this sequence, ORFs were identified using start and stop codons on both strands for the region including base pairs 1-863 of Ad5, the E. coli fragment, and the beginning of the predicted sequence of NheI-linearized pAdCMVhB7-1. A total of 13 ORFs were identified in this region, ranging in size from 25 to 296 amino acids (aa) in length. Three of these ORFs are unique to this construct because they span the junction between either base pairs 1-863 of Ad5 and the E. coli fragment or the E. coli fragment and the predicted sequence of NheIlinearized pAdCMVhB7-1. On the plus strand, ORF1 begins with the start codon for E1a, continues through the first 101 aa of E1a, and extends for an additional 92 aa into the $E$. coli fragment. On the minus strand, ORF2 begins with the start codon and first three amino acids in the predicted sequence of NheI-linearized pAdCMVhB7-1 and extends for 293 aa into the $E$. coli fragment. This ORF includes a hypothetical but innominate protein that is identified in the GenBank entry for E. coli minutes 9-11 (GenBank accession number U82664). Also on the minus strand, ORF3 begins with the start codon and first 104 aa of a protein identified in the GenBank database as similar to the carbamate kinase of Pseudomonas aeruginosa and extends an additional 22 aa into base pairs 1-863 of Ad5. 


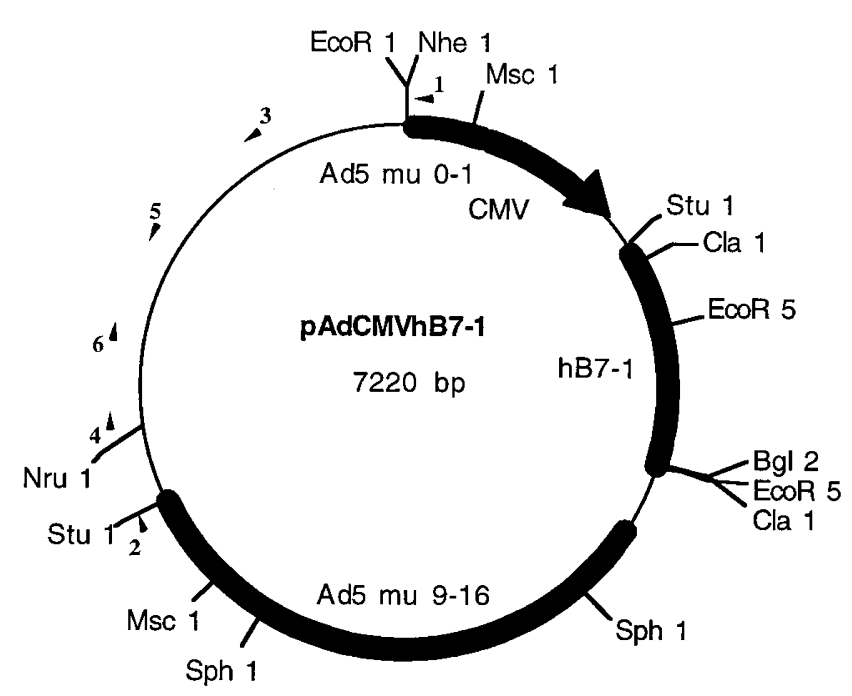

Figure 3. A plasmid map of pAdCMVhB7-1 indicating sequencing primers (arrows, 1-6) and restriction digestion sites. Ad5 mu 0-1, base pairs 1-352 of adenovirus 5; CMV, CMV immediate early enhancer/promoter; hB7-1, hB7-1 cDNA; and Ad5 mu 9-16, base pairs 3329-5792 of adenovirus 5 . Primers used to sequence pAdCMVhB7-1 are: 1, 5'-CCCCCTCATTATCATATTGGCTTC-3'; 2, 5'-TGACGAAAAGGCTGTCCGTG-3'; 3, 5'-GCCAACTTACTTCTGACAACG-3'; 4, 5'-CCCCATTATGATTCTTCTCG-3'; 5, 5'-GACCAAAATCCCTTAACGTG-3'; and 6, 5'-GTATCTCAGTTCGGTGTAGGTC-3'.

$P C R$, sequence, and Southern blot analysis of pAdCMVhB7-1 for the presence of the E. coli fragment. We hypothesized that the E. coli fragment found in Ad.ihB7-1 immediately upstream of the ITR of pAd-CMVhB7-1 (see Fig 2) originated from $E$. coli genomic DNA contaminating large-scale preparations of pAd-CMVhB7-1. We designed primers based on the sequence of Ad.ihB7-1 (positions are indicated in Fig 2) to detect this fragment in the plasmid stock. We tested the plasmid from which pAd-CMVhB7-1 was derived (pAd-CMVlink) and a number of plasmids based on pAd-CMVlink. Using the same PCR reaction, we also tested the components of the cotransfection experiment that were used to create Ad.ihB7-1. The E. coli DNA sequence could not be detected by PCR in the 293 cell genomic DNA, salmon sperm DNA, $N$-2-hydroxyethylpiperazine- $N^{\prime}$-2-ethanesulfonic acid-buffered saline, $\mathrm{CaCl}_{2}$, or ClaI-digested dl7001 DNA. However, all of the plasmid stocks of pAdCMVhB7-1 tested contained this fragment, as indicated by the 404-bp product obtained (data not shown).

Next, we partially sequenced pAdCMVhB7-1 to localize the $E$. coli fragment. The locations of the sequencing primers are indicated in Figure 3. The E. coli fragment that is present in Ad.ihB7-1 was not located in the sequenced region of pAdCMVhB7-1, and restriction digestions showed that it is also not present in the unsequenced part of pAdCMVhB7-1 (data not shown).

The E. coli fragment was not located through the sequencing of pAdCMVhB7-1; therefore, we attempted to localize this fragment through Southern blot analysis. We used a randomly labeled 500-bp PCR product of this
Table 1. Assay for Replicative Ability

\begin{tabular}{|c|c|c|c|c|c|c|}
\hline \multicolumn{2}{|c|}{ Infection 1} & \multicolumn{2}{|c|}{ Infection 2} & \multicolumn{3}{|c|}{ Infection 3} \\
\hline Input & CPEFlow & Input & CPEFlow & Input & $\mathrm{CPE}$ & Flow \\
\hline Ad.lacZ & $-100 \%$ & $0 \lambda$ lysate & $-93 \%$ & $0 \lambda$ lysate & - & $0 \%$ \\
\hline Ad.inB7-1 & $+90 \%$ & $0 \lambda$ lysate & $-14 \%$ & $0 \lambda$ lysate & - & $0 \%$ \\
\hline dl7001 & $+\quad N A$ & $0 \lambda$ lysate & $+\mathrm{NA}$ & $50 \lambda$ lysate & + & NA \\
\hline
\end{tabular}

NA, not applicable.

region in Ad.ihB7-1 as a probe. Despite effective labeling of the positive control, only faint bands in lanes containing plasmid DNA were detectable (data not shown). This indicates that the E. coli fragment is not incorporated into pAdCMVhB7-1. Therefore, E. coli genomic DNA contaminating the plasmid stock appears to be the source of the E. coli DNA fragment, which was subsequently incorporated into Ad.ihB7-1.

\section{Characterization of replicative ability}

The ability of Ad.ihB7-1 to replicate in nontranscomplementary (E1-negative) cell lines was assessed in HeLa cells. Replication can be detected qualitatively by the ability of lysate from initially transfected cells to transduce a second population of cells. If Ad.ihB7-1 is replication-competent (by incorporation of a functional E1), lysate from cells infected with Ad.ihB7-1 should be able to transduce other cells to express $B 7-1$. The ability of Ad.ihB7-1 to replicate was compared with dl7001 (which is known to be replication-competent) and Ad.lacZ (which is E1-deleted and free of RCAs).

Two plates of HeLa cells were each infected at an MOI of 50 based on plaque-forming units with dl7001, Ad.lacZ, or Ad.ihB7-1. One plate infected with each virus was observed for the development of cytopathic effect (CPE) over 3 days, whereas the second plate was assessed at 72 hours by flow cytometry for the presence of transgene (either $\beta$-gal or hB7-1). HeLa cells infected with dl7001 or Ad.ihB7-1 developed a complete CPE within 48 hours, whereas cells infected at the same MOI with nonreplicating Ad.lacZ did not develop CPE. Both hB7-1 and $\beta$-gal were detectable by flow cytometry (Table 1).

Lysates were created by repeated freeze/thawing of transduced HeLa cells and were used to transduce two sets of plates of fresh HeLa cells. These new HeLa cells were treated as described above. Only those cells infected with dl7001 lysate developed CPE. Cells infected with lysates containing either Ad.lacZ or Ad.ihB7-1 did, however, express transgene protein that was detectable by flow cytometry (Table 1 ).

Lysates from the second round of infection were used to infect two sets of plates of fresh HeLa cells and were treated as described above. Again, cells infected with dl7001 lysate were the only ones to develop CPE, and cells infected with lysate containing Ad.lacZ or Ad.ihB7-1 did not have detectable levels of transgene expression at this stage (Table 1). These results indicate 
that Ad.ihB7-1 is not fully replication-competent, despite the presence of a portion of the E1a ORF.

Competition assay to detect a growth advantage of Ad.ihB $7-1$

We hypothesized that the incorporation of additional DNA, including part of E1a, could impart a growth advantage to Ad.ihB7-1 compared with the desired rAd (Ad.hB7-1). This potential growth advantage could be due to the expression of one of the unique ORFs, particularly ORF1, which contains part of E1a, or due to the more efficient packaging of a genome size closer to that of wt Ad5. The Ad.ihB7-1 genome is $34 \mathrm{~kb}$ or $\sim 95 \%$ of the Ad5 genome. In contrast, the Ad.hB7-1 genome that we were attempting to construct is $32 \mathrm{~kb}$ or $89 \%$ of the Ad5 genome. We compared the growth characteristics of Ad.ihB7-1 and Ad.mB7-1 in 293 cells using a competition assay. Ad.mB7-1 differs from Ad.hB7-1 by only 24 bp. Thus, Ad.mB7-1 is a suitable substitute for Ad.hB7-1 in comparison with Ad.ihB7-1. Initially, Ad.mB7-1 and Ad.ihB7-1 genomic DNA was precipitated into 293 cells in duplicate in molar ratios of 10:1, 1:1, and 1:10 (320 ng of total DNA per precipitation). This experiment was then repeated with $2.2 \mu \mathrm{g}$ of total DNA per precipitation. The noncellular DNA isolated at the time of CPE was then analyzed by Southern blot analysis. Quantification of the PhosphorImager data acquired from these experiments showed no significant difference between the ratios of input and output virus (data not shown). Therefore, no growth advantage for Ad.ihB7-1 was seen.

\section{DISCUSSION}

We have characterized an aberrant viral construct that arose during the construction of an E1, E3-deleted rAd expressing the hB7-1 gene. Ad.ihB7-1 produced functional transgene as indicated by a T-cell costimulation assay and flow cytometric analysis of transduced cells. However, restriction analysis showed an insertion of additional DNA upstream of the transgene. More detailed sequence analysis led to the identification of all elements comprising this upstream insertion.

This viral construct is unusual because it seems to be the result of both homologous and nonhomologous recombination events. The initial ITR and portion of E1a that are present in Ad.ihB7-1 were most likely derived from the 293 cells, because no other source of E1a exists among the elements of the original cotransfection. Lochmuller et al detected the emergence of E1-positive RCA in originally pure E1-negative/E3negative rAd stocks after serial passage on 293 cells. They postulated that the RCA was the result of recombination between rAds and adenoviral sequences in the 293 genome. $^{7}$ A similar homologous recombination event is described by Hehir et al; ${ }^{17}$ however, the resultant RCAs were Ad2/Ad5 chimeras, allowing the origin of each fragment to be determined by sequence analysis.

The addition of a portion of an E. coli genome and its position relative to the other components of Ad.ihB7-1 is the most striking feature of the vector construct. We hypothesized that this E. coli DNA originated from contaminating genomic bacterial DNA present in the plasmid stock used to generate Ad.ihB7-1. Detailed restriction mapping and sequencing of the majority of pAdCMVhB7-1 did not reveal the presence of this sequence. The map of pAdCMVlink, the plasmid from which pAdCMVhB7-1 is derived, also does not contain this $E$. coli genome sequence. We have analyzed preparations of pAdCMVlink and a number of plasmids based on pAdCMVlink, including pAdCMVhB7-1, by PCR for the presence of this $E$. coli fragment and have found this fragment in pAdCMVlink and all plasmids derived from pAdCMVlink. Southern blot analysis of pAdCMVhB7-1 using a PCR product from Ad.ihB7-1 as a probe revealed minimal hybridization of the probe to plasmid DNA. Taken together, these data indicate that the $E$. coli fragment is present as contaminating $E$. coli genomic DNA in all preparations of plasmid DNA and is detectable by sensitive PCR methods. Southern blot analysis, restriction digest, and sequencing fail to detect this sequence, because it is not incorporated into the plasmid DNA and is present in trace quantities. We recognize that this DNA could be a contaminant of any of the components of the cell system used to generate Ad.ihB7-1; however, the components of the cotransfection (salmon sperm DNA, dl7001, N-2-hydroxyethylpiperazine- $N$ '-2-ethanesulfonic acid-buffered saline, $\mathrm{CaCl}_{2}$, and 293 cell DNA) were all negative when tested by PCR. Thus, this sequence most likely originated in the plasmid stock of pAdCMVhB7-1 and was incorporated into the vector through nonhomologous recombination.

Other groups have reported the isolation of unanticipated mutant constructs that arose during the formation of rAds. Haj-Ahmad and Graham ${ }^{18}$ characterized a rAd based on Ad5 that arose during the construction of an E1, E3 deletion mutant. This mutant (dlE1,3-1) consisted of a second copy of $2.6 \%$ of the $5^{\prime}$ end of the Ad5 genome attached to a portion of pBR322, which was then linked to the $5^{\prime}$ end of the desired rAd. During propagation, this additional segment transferred to the $3^{\prime}$ end of the genome. The resulting mutant (dlE1,3-2) with additional segments on both ends of the genome reverted with high frequency back to dlE1,3-1. The mechanism by which dlE1,3-1 arose was explained by a combination of homologous recombination and in vivo ligation of the plasmid and genomic components of the cotransfection that were digested with ClaI ${ }^{18}$

Hehir et al reported the characterization of an RCA that arose through homologous recombination between the rAd they were trying to construct and the 293 cellular genome. ${ }^{17}$ Through detailed sequence analysis, they were able to confirm that three RCAs consisted of Ad2/Ad5 chimeras with Ad2 sequences flanking 2.6-3.8 $\mathrm{kb}$ of Ad5 DNA. The homologous recombination events that resulted in these RCAs restored the E1 sequences derived from the 293 cellular genome (which contains Ad5 DNA) in an Ad2-based rAd, rendering it replication-competent.

The previously published constructs described above 
could be attributed to defined homologous recombination events. In contrast, Ad.ihB7-1 seems to have resulted from nonhomologous recombination events. Nonhomologous recombination between adenovirus DNA and cellular DNA has been described for Ad12 and Ad5. Deuring et al describe an Ad12/cellular DNA chimera (symmetric recombinant 1) that consists almost entirely of cellular DNA flanked by 700-1150 bp of the 5' end of the Ad12 genome in a large inverted repeat of the type $\mathrm{ABCDD}^{\prime} \mathrm{C}^{\prime} \mathrm{B}^{\prime} \mathrm{A}^{\prime} .{ }^{19}$ This construct was stable through serial passage for years. Further analysis of a second construct (symmetric recombinant 2) revealed that 2081 bp of the left terminus of Ad12 were linked directly to cellular DNA. ${ }^{20}$ Interestingly, this additional DNA extended the ORF of E1b for an additional 66 aa, although the resulting hypothetical protein was not isolated.

The appearance of Ad.ihB7-1 instead of Ad.hB7-1 seems unlikely, given the number of recombination events that must occur. Therefore, we hypothesized that a rare event was amplified by a growth advantage for the virus with a genome size closer to that of wt Ad5 and containing part of E1a. However, the competition assay indicates no significant growth advantage for Ad.ihB7-1 over Ad.mB7-1. Despite the lack of any apparent advantage for the incorporation of additional DNA in a rAd of this size, we have also isolated Ad.mB7-1 recombinants that contain a functional mouse $B 7-1$ gene and that also include additional DNA ( $\leq 2 \mathrm{~kb})$ at the $5^{\prime}$ end. However, the other three hB7-1-positive plaques from the initial cotransfection of pAdCMVhB7-1 and dl7001 have the correct Ad.hB7-1 sequence.

The appearance of Ad.ihB7-1 highlights the need for careful analysis of rAd stocks constructed for clinical use. Despite functional analysis indicating that Ad.ihB7-1 was able to function in the manner intended, detailed sequence analysis indicated the unusual structure that has been described. Sequencing of the "minigene cassette" (promoter/enhancer, transgene, and polyadenylation signal) alone would not reveal this abnormality. Although Ad.ihB7-1 seems to be replication-defective, the consequences of the arrangement of the constitutive fragments and the extension of the E1a ORF are not known. rAd stocks must be carefully analyzed before clinical use to detect subpopulations of RCAs and rearranged rAds.

\section{ACKNOWLEDGMENTS}

We thank Dr. Paul Bates for his thoughtful review of the manuscript and Dr. Heike Boxhorn for her technical support. This work was supported by US Public Health Service Grants PO1-CA-25874, UO1-CA-65805, and RO1-CA-67776 from the National Cancer Institute.

\section{REFERENCES}

1. McGarrity G. Recent advances in gene therapy. GEN Guide to Biotechnology Companies. 1995:78-79.
2. Jolly D. Viral vector systems for gene therapy. Cancer Gene Ther. 1994;1:51-64.

3. Berkner K. Development of adenovirus vectors for the expression of heterologous genes. Biotechniques. 1988;6: $616-629$.

4. Kozarsky KF, Wilson JM. Gene therapy: adenovirus vectors. Curr Opin Genet Dev. 1993;3:499-503.

5. Wang Q, Finer MH. Second-generation adenovirus vectors. Nat Med. 1996;2:714-716.

6. Weitzman MD, Wilson JM, Eck SL. Adenovirus vectors in cancer gene therapy. In: Sobol RE, Scanlon KJ, eds. The Internet Book of Gene Therapy: Cancer Therapeutics. Stamford, Conn: Appleton and Lange; 1995:17-25.

7. Lochmuller H, Jani A, Huard J, et al. Emergence of early region 1-containing replication-competent adenovirus in stocks of replication-defective adenovirus recombinants $(\Delta \mathrm{E} 1+\Delta \mathrm{E} 3)$ during multiple passages in 293 cells. Hum Gene Ther. 1994;5:1485-1491.

8. Epstein LH, Young CS. Adenovirus homologous recombination does not require expression of the immediateearly E1a gene. J Virol. 1991;65:4475-4479.

9. Graham FL, Smiley J, Russell WC, Nairn R. Characteristics of a human cell line transformed by DNA from human adenovirus type 5. J Gen Virol. 1977;36:59-74.

10. Louis N, Evelegh C, Graham FL. Cloning and sequencing of the cellular-viral junctions from the human adenovirus type 5 transformed 293 cell line. Virology. 1997;233:423-429.

11. Bett AJ, Prevec L, Graham FL. Packaging capacity and stability of human adenovirus type 5 vectors. $J$ Virol. 1993;67:5911-5921.

12. Parks RJ, Graham FL. A helper-dependent system for adenovirus vector production helps define a lower limit for efficient DNA packaging. J Virol. 1997;71:3293-3298.

13. Ye X, Robinson M, Batshaw M, Furth E, Smith I, Wilson J. Prolonged metabolic correction in adult ornithine transcarbamylase-deficient mice with adenoviral vectors. J Biol Chem. 1996;271:3639-3646.

14. Ranheim TS, Shisler J, Horton TM, Wold LJ, Gooding LR, Wold WS. Characterization of mutants within the gene for the adenovirus E3 14.7-kilodalton protein which prevents cytolysis by tumor necrosis factor. J Virol. 1993; 67:2159-2167.

15. Logan J, Shenk T. Adenovirus tripartite leader sequence enhances translation of mRNAs late after infection. Proc Natl Acad Sci USA. 1984;81:3655-3659.

16. Boxhorn HKE, Smith JG, Chang Y, et al. Adenoviral transduction of melanoma cells with B7-1: antitumor immunity and immunosuppressive factors. Cancer Immunol Immunother. 1998;46:283-292.

17. Hehir KM, Armentano D, Cardoza LM, et al. Molecular characterization of replication-competent variants of adenovirus vectors and genome modifications to prevent their occurrence. J Virol. 1996;70:8459-8467.

18. Haj-Ahmad Y, Graham FL. Characterization of an adenovirus type 5 mutant carrying embedded inverted terminal repeats. Virology. 1986;153:22-34.

19. Deuring R, Klotz G, Doerfler W. An unusual symmetric recombinant between adenovirus type 12 DNA and human cell DNA. Proc Natl Acad Sci USA. 1981;78:3142-3146.

20. Deuring R, Doerfler W. Proof of recombination between viral and cellular genomes in human $\mathrm{KB}$ cells productively infected by adenovirus type 12: structure of the junction site in a symmetric recombinant (SYREC). Gene. 1983;26: 283-289. 